\title{
Calculation of Eddy Current Loss and Short-circuit Force in SSZ11-50000/110 Power Transformer
}

\author{
Yan Li ${ }^{1}$, Bo Zhang ${ }^{1}$, Longnv Li ${ }^{1}$, Tongxun Yang ${ }^{2}$, Ning Wang ${ }^{2}$ \\ ${ }^{1}$ Power Transmission Technology Research Institution, Shenyang University of Technology, Shenyang \\ ${ }^{2}$ TBEA Shenyang Transformer Group Co., Ltd. Shenyang \\ Email: solomon.zb@163.com
}

Received April, 2013

\begin{abstract}
The $T-\Omega$ method is used in this paper to calculate the leakage magnetic field of SSZ11-50000/110 Power transformer, and by which the structures' influences to the main leakage flux are analyzed. Through the combination of the product and TEAM Problem 21B, the surface impedance method shows its great advantage in the calculation of eddy current loss.
\end{abstract}

Keywords: Power Transformer; Finite Element; Short-circuit Force; Eddy Current Loss; Surface Impedance Method

\section{Introduction}

With the rising of voltage level and transformer capacity, the leakage flux of transformer increased, therefore, the problem of structural part stray loss and short-circuit strength has became one of urgent key technical issues both in transformer companies and operation department.

To solve this type of problem, intra-industry corresponding standards are already existed, and it changes with the products upgrade.

The traditional analytical method cannot analysis the local overheating, short circuit mechanical force withstand capability and three-dimensional spatial distribution of leakage flux which the new standards request. Based on three-dimensional finite element numerical method, the paper introduced transformer design criterion to discuss the leakage flux distribution, short circuit strength, and eddy current loss of transformer, and the fitted engineering design loss calculation numerical method is also proposed [1,2].

\section{Mathematical Model}

The $T-\Omega$ method which uses scalar magnetic potential and electric vector potential can reduce unknown number and save computer memory when high precision is required.

The governing equations in eddy zone is

$$
\nabla \times\left[\left(\sigma+\varepsilon \frac{\partial}{\partial \mathrm{t}}\right)^{-1} \nabla \times \mathrm{T}\right]+\mu \frac{\partial}{\partial t}[(\mathrm{~T}-\nabla \Omega)]=0
$$

\footnotetext{
*This work was supported by NSFC, under Project 51177103 and Program for LNIRT in University (LT2011002).
}

penalty function is introduced,

$$
\begin{aligned}
-\nabla\left(\sigma+\varepsilon \frac{\partial}{\partial t}\right)^{-1} \nabla \cdot \mathrm{T}, \text { then } \\
\nabla \times\left[\left(\sigma+\varepsilon \frac{\partial}{\partial t}\right)^{-1} \nabla \times \mathrm{T}\right]-\nabla\left(\sigma+\varepsilon \frac{\partial}{\partial t}\right)^{-1} \nabla \cdot \mathrm{T} \\
+\mu \frac{\partial}{\partial t}[(\mathrm{~T}-\nabla \Omega)]=0
\end{aligned}
$$

$\nabla \cdot B=0$, then

$$
\nabla \cdot[\mu(T-\nabla \Omega)]=0
$$

The governing equations in non-eddy zone is

$$
\nabla \cdot\left[\mu\left(-\nabla \Omega+\mathrm{H}_{s}\right)\right]=0
$$

where, Hs is the magnetic field produced by source current in infinite space, $\mathrm{T}$ is electric vector potential, $\Omega$ is scalar magnetic potential, $\sigma$ is conductivity, $\varepsilon$ is dielectric constant, $\mu$ is permeability.

The calculation process is treated as follows:

1) According to structural symmetry, the half transformer model is taken into calculation (as shown in Figure 1);

2) Circulation and eddy current in winding, and eddy current in core is neglected;

Using wire winding, and the current density is uniform in the winding.

$$
J=\frac{N}{S} I
$$

where $J$ is winding current density, $N$ is winding turns, $S$ is sectional area, $I$ is winding current; 
3) Material of iron core is according to rolling direction and orthogonal opposite treated. The material of tank, draw plate and clamping part are treated according to each direction same treated. The basic magnetization characteristic curve is used here and hysteresis characteristic is neglected.

4) The Newton-Raphson algorithm is used to solve the magnetization characteristic of core silicon steel sheet and tank steel plate for their characteristic are nonlinear relation.

5) The eddy zone produce skin effect, and mesh generation should take current density radial distance.

$$
\delta=\sqrt{\frac{1}{\pi f \sigma \mu}}
$$

where $\delta$ is penetration depth, $f$ is frequency, $\sigma$ is conductivity, $\mu$ is permeability. According to field value some gradient descent discipline to stratify, penetration depth chooses $0.22,0.51,0.92,1.6$ and 5 times to mesh generation.

\section{Field Analysis}

To analysis short-circuit electromagnetic force, the flux leakage magnetic distribution should be studied first.

As shown in Figure 2 that winding average flux density along circumferential distribution, winding end magnetic density is affected by upper and lower iron yok, the inner magnetic density is higher than the outers'. The radiation component $\mathrm{Br}$ value is impacted greatly from $24.35 \mathrm{mT}$ to $27.8 \mathrm{mT}$. The axis component $\mathrm{Bz}$ value varies from $58.4 \mathrm{mT}$ to $61.6 \mathrm{mT}$, and both of their two sides are more than that at the middle part. The middle of the winding does not change greatly for its far distant form clamping part and iron yoke.

For the anglicizing of the structure eddy current loss, the surface field and eddy current distribution are the emphases.

As shown in Figure 3 that the magnetic curve of outer tank side winding end part is bend, and the vortex center is formed for the leakage flux concentrated into the tank surface.

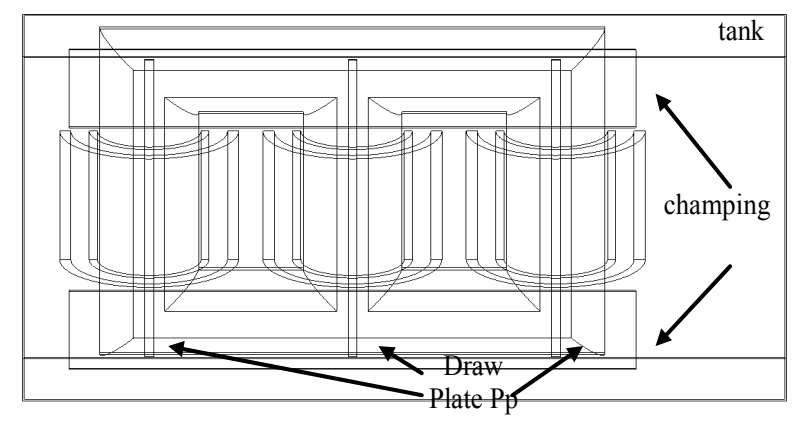

Figure 1. Transformer calculation model.
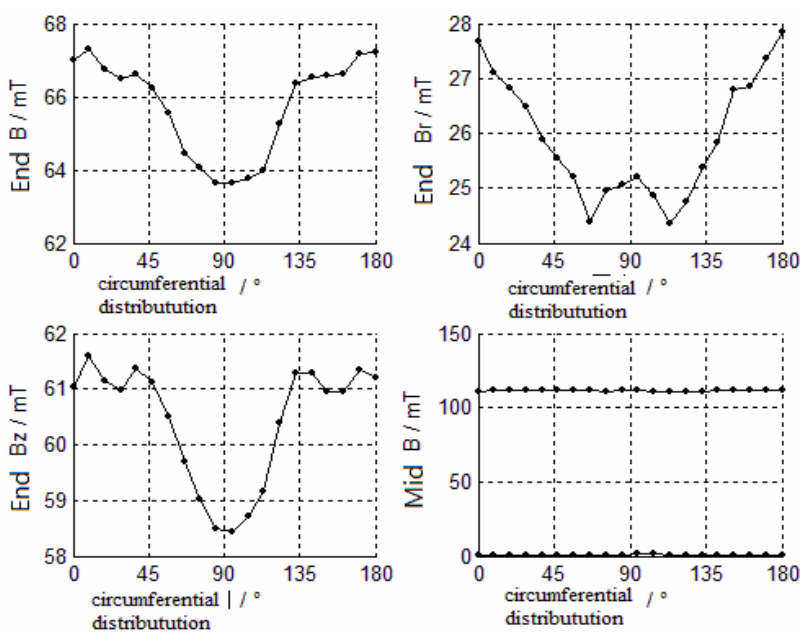

Figure 2. Winding average flux density along circumferential distribution.

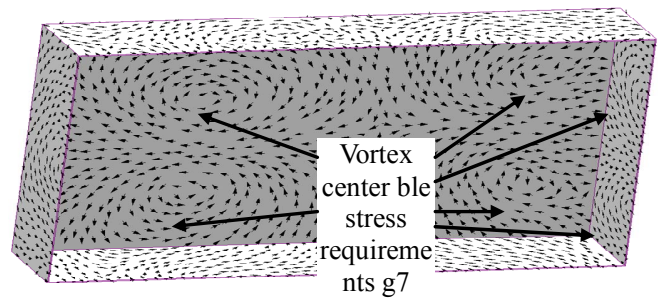

Figure 3. The surface of the oil tank eddy current distribution.

According to the CIGRE recommended document, the transformer metal structure of local overheating criteria, through calculation, the tank surface magnetic field strength of the tangential component maximum value was $48.22 \mathrm{~A} \cdot \mathrm{cm}^{-1}$, does not exceed the standard value of $60 \mathrm{~A} \cdot \mathrm{cm}^{-1}$, therefore, the additional fuel tank shield is not necessary.

\section{Short Circuit Strength}

The national standard GB1094.5-2008, in the new version, the allowable stress requirements of .conventional, non - viscous, sticky wire and CTCs (continuous transposed conductor) are different from the original design.

Now, take average ring stress $\sigma_{\mathrm{t} 1}$, warping free limit stress $\sigma_{\text {cr }}$, the inner winding radial bending stress $\sigma_{\text {sav }}$, axial compressive stress $\sigma_{\text {act }}$, conductor axial bending stress $\sigma_{A L}$, and wire inclination critical stress $\sigma_{c r_{-} \text {tilt }}$ for example $[3,4]$

According to the national standard GB1094.2-2008, the first peak of asymmetric short-circuit current is

$$
I_{\text {峰 }}=\frac{i \times k \times \sqrt{2}}{U_{k}}
$$

where, $i$ is rated current effective value, $U_{k}$ is short circuit impedance, $k \times \sqrt{2}$ is peak factor, in this paper 
the transformer is valued 2.55.

According to the Lorenz formula, the winding electromagnetic force can be calculated., The SSZ11-50000/ 110 transformer short-circuit force calculated values and national standard GB1094.5-2008 requirements shortcircuit mechanical strength are shown in Table 1.

Through calculation, the low-voltage windings of power transformer SSZ11-50000/110 meet the national standard GB1094.5-2008 to withstand short-circuit mechanical strength requirements.

\section{Eddy Current Loss}

Eddy current density can be calculated by the following formula

$$
\boldsymbol{J}_{\mathrm{e}}=-\sigma\left(\frac{\partial \boldsymbol{A}}{\partial t}+\Delta V\right)
$$

Eddy current loss

$$
p_{\mathrm{e}}=\int_{v} \frac{\left|\mathbf{J}_{\mathrm{e}}\right|^{2}}{\sigma} d v
$$

where, Je is eddy current density, pe is Eddy current loss, Ais magnetic vector potential, $\mathrm{V}$ is electric scalar potential.

As shown in Figure 4, the maximum point appears in the main empty path up(down) direction, base on design experience, the structural overheating criterion is shown in Table 2

Table 1. SSZ11-50000/110 anti short-circuit stability.

\begin{tabular}{lccc}
\hline & $\sigma_{\mathrm{t} 1}\left(\mathrm{MP}_{\mathrm{a}}\right)$ & $\sigma_{\mathrm{cr}}\left(\mathrm{MP}_{\mathrm{a}}\right)$ & $\sigma_{\text {sav }}\left(\mathrm{MP}_{\mathrm{a}}\right)$ \\
\hline Calculation value & 38.24 & 11.70 & 66.01 \\
National standard & $\leq 49$ & $\leq 98$ & $\leq 126$ \\
& $\sigma_{\text {act }}\left(\mathrm{MP}_{\mathrm{a}}\right)$ & $\sigma_{A L}\left(\mathrm{MP}_{\mathrm{a}}\right)$ & $\sigma_{c r_{-} \text {tilt }}\left(\mathrm{MP}_{\mathrm{a}}\right)$ \\
Calculation value & 50 & 75.51 & 28.32 \\
National standard & $\leq 80$ & $\leq 126$ & $\leq 98$ \\
\hline
\end{tabular}

\section{outside}

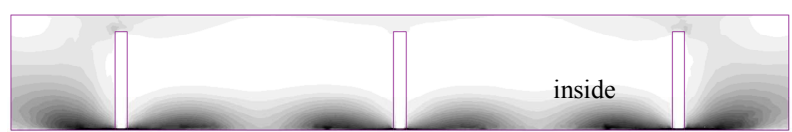

Figure 4. The eddy current loss density distribution of Clamp part.

Table 2. Large transformer partial overheat judgment.

\begin{tabular}{cc}
\hline Structure part & maximum loss density $/ \mathrm{kW} \cdot \mathrm{m}^{-3}$ \\
\hline tank & 1800 \\
champ & 1800 \\
\hline
\end{tabular}

Through calculation, the highest tank eddy current loss density is $1066.3 \mathrm{~kW} \cdot \mathrm{m}^{-3}$. And the clamp part maximum loss density is $2100.5 \mathrm{~kW} \cdot \mathrm{M}^{-3}$, which exceed the empirical criterion. Through taking effective measures, the possible overheating is prevented.

\section{Surface Impedance Method}

When eddy current loss in transformer is under calculation, if more structures exists namely the vortex area accounts for a larger proportion of the solution domain, it will lead to a large amount of computer memory occupied.

After taking into the consideration of the skin effect, the refinement of mesh in the penetration depth is also needed. Even using hierarchical subdivision method, a smaller grid is still needed to meet the needs of accuracy.

Taking TEAM Problem 21B as an example [5], the relations of steel plate maximum length and the calculated value eddy current loss is shown as Figure 5.

When the maximum length of the element is $5 \mathrm{~mm}$, it will cost one hour to reach the experimental values to meet the precision need, and the method can not be applied in the calculation model because the structure size growth several times which makes the calculation up to tens of hours.

When the skin depth is small, the surface of eddy region is regarded as the impedance boundary conditions for solving domain, and the finite element method are still used in other domains that can obviously reduce the problem scale.

The surface impedance eddy zone definition

$$
Z_{s}=\frac{E_{t}}{H_{t}}=(1+j) \sqrt{\frac{\pi f \mu}{\sigma}}=\frac{1+j}{\delta \sigma}
$$

where $Z_{s}$ is surface impedance, $E_{t}$ is tangential component of electric field intensity, $H_{t}$ is tangential component of magnetic field intensity, $\delta$ is penetration depth.

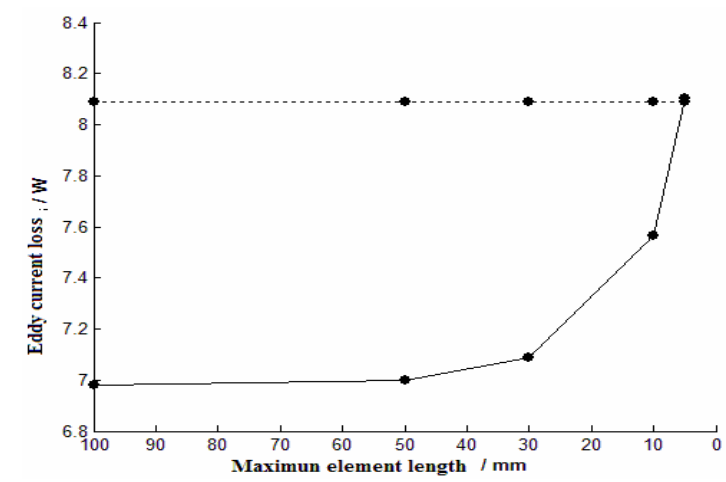

Solid line: the calculated values; dotted line: measured value

Figure 5. 21B relations of maximum element length and calculation value. 
Table 3. Eddy current loss calculation.

\begin{tabular}{ccccc}
\hline Unit: $\mathrm{W}$ & \multicolumn{2}{c}{ SSZ11-50000/110 transformer } & problem21B \\
\hline \multirow{2}{*}{ Calculation value } & tank & champ & draw & champ \\
& 9853.9 & 13054.57 & 4369.9 & 8.14 \\
Measured value & & & & 8.09 \\
\hline
\end{tabular}

After using surface impedance method, the calculation time has been significantly decreased, and the eddy current loss calculation is shown in Table 3.

\section{Conclusions}

The $T-\Omega$ method is used in this paper to calculate the parameters of SSZ11-50000/110 transformer, and the conclusion can be summarized as follows:

1) The short-circuit mechanical strength meets the new requirements; the tangential component of structure surface field is lower than the local overheating criterion. According to the design experience, surface eddy current density of the clamp part greatest is too high, and there is a risk of local overheating, for which the shielding equipment is considered installing in the clamps.

2) The end winding leakage flux along the circumfer- ential distribution is not uniform for the effect of iron yoke. The calculation of the winding end axial magnetic flux density is influenced by structure and should not be ignored when the short-circuit force is calculated.

3) By using hierarchical subdivision method for the calculation of transformer structure eddy current losses is time-consuming and difficult to ensure the accuracy. And using the finite element method of surface impedance boundary can solve this problem effectively.

\section{REFERENCES}

[1] “Transformer design principle of," China Electric Power Press.

[2] C. Y. Liu, "Calculation Method and Practice of Transformer Design," Liaoning Science and Technology Press.

[3] J. Liu and A. H. Zhang, "Transformer Simulation and Evaluation of Power Winding Short-circuit Dynamic Stability," Transformer, Vol. 49, No. 6, 2012, pp.14-25.

[4] GB1094.5-2008. Requirements Short-circuit Mechanical Strength

[5] S. M. Yang, Z. G. Cheng and X. Q. Zhu, "The Stray Loss in Steel Measurement Technology," Chinese Journal of Scientific Instrument. 